\title{
All-Polyethylene Tibial Component Does Not Affect Survivorship of Medial Unicompartmental Knee Arthroplasty at Mid-Term Follow-Up
}

\author{
Michele Gagliardi, MD ${ }^{1}$ Francesco Zambianchi, MD ${ }^{1}$ 잉 \\ Fabio Catani, $\mathrm{MD}^{1}$ \\ ${ }^{1}$ Department of Orthopaedic Surgery, Università degli Studi di \\ Modena e Reggio Emilia, Modena, Emilia-Romagna, Italy \\ 2 Department of Orthopedic Surgery and Sports Traumatology, \\ St. Marien-Krankenhaus - Siegen, Germany \\ 3 1st Orthopedic Clinic, Istituto Ortopedico Rizzoli, Bologna, \\ Emilia-Romagna, Italy
}

J Knee Surg 2021;34:1454-1462.

\author{
Alois Franz, MD² Vitantonio Digennaro, $\mathrm{MD}^{3}$
}

Address for correspondence Francesco Zambianchi, MD, Department of Orthopaedic Surgery, Università degli Studi di Modena e Reggio Emilia, Modena, Emilia-Romagna, Italy (e-mail: francesco.zambianchi@gmail.com).

\begin{abstract}
The present study's primary aim was to determine the survivorship of a large cohort of patients implanted with a single design all-polyethylene tibial component medial unicompartmental knee arthroplasty (UKA). Its secondary purpose was to investigate the reasons underlying implant failure, with specific attention to component positioning and limb alignment. Between 2007 and 2013, 166 patients underwent medial UKA with a single design all-polyethylene tibial component at two centers. Preoperatively and postoperatively, patients were administered clinical outcome scores and radiographic information were collected. Postoperative complications and causes of revision were recorded. A total of 140 patients (80 in Center A and 60 in Center B) who underwent all-polyethylene tibial component medial UKA ( 82 cases in Center $A$ and 60 in Center B) were taken into account. Kaplan-Meier cumulative survivorship of implants was $96.5 \%$ (confidence interval [Cl]: 91.7-98.6\%) at an average follow-up of 61.1 months. Tibial aseptic loosening was accounted for failure in one case, while no

\section{Keywords}

- all-polyethylene

- unicompartmental knee arthroplasty

- survivorship

- alignment correlation was found between implant positioning and failure. Two revisions were performed in Center A and three in Center B. Slight correction of the preoperative varus deformity was performed at both centers. All-polyethylene tibial component UKA provided satisfactory clinical and functional outcome, with excellent survival rate in the early and mid-term follow-up. Continued patient follow-up is needed to determine long-term survivorship of the examined UKA model.
\end{abstract}

The superiority of an all-polyethylene or a metal-backed tibial component in unicompartmental knee arthroplasty (UKA) for the treatment of knee osteoarthritis (OA) or osteonecrosis continues to be a point of debate. Some authors have reported reduced survival rates, poorer functional outcomes, and increased difficulties in intraoperative alignment with all-polyethylene UKAs compared with metal-backed implants, ${ }^{1-3}$ whereas others ${ }^{4-6}$ have demonstrated satisfactory outcomes

received

January 15, 2019

accepted

March 21, 2020

published online

May 25, 2020

and excellent survivorship with all-polyethylene prostheses. Despite the reduced costs and the theoretically improved wear characteristics favoring the use of all-polyethylene tibial components in UKA, it remains unclear whether the clinical outcomes and survivorship rates are comparable to metalbacked modular components. ${ }^{7}$

Only a few authors have assessed survivorship rates and clinical performance of UKA using an all-polyethylene tibial

(c) 2020. Thieme. All rights reserved. Thieme Medical Publishers, Inc., 333 Seventh Avenue, 18th Floor, New York, NY 10001, USA
DOI https://doi.org/ 10.1055/s-0040-1710360. ISSN 1538-8506. 
component at long-term follow-up. Heyse et $\mathrm{al}^{8}$ reported a survivorship of approximately $93 \%$ at 10.8 years in a nonhomogeneous series of UKAs including medial and lateral components with both all-polyethylene and metal-backed tibial implants. At a mean follow-up of 10 years, Lustig et $\mathrm{al}^{6}$ reported an overall survivorship of approximately $93 \%$ in allpolyethylene implants including medial and lateral UKAs. Recent papers report survival probability higher than 95\% at a mean follow-up of 8 years, which is comparable to reports from studies using metal-backed UKAs. ${ }^{9}$ Other studies have highlighted early failures in all-polyethylene UKAs, particularly in obese patients and in those with significant bony defects. $^{10,11}$

There are still differing interpretations about the correct placement and alignment of the prosthetic components relative to the design. ${ }^{12,13}$ Nevertheless, adequate limb and prosthetic components' alignment are considered to lead to satisfactory outcomes, no matter what tibial component is selected. ${ }^{6}$ In general, a slight hypocorrection of the preoperative varus limb deformity and some residual degrees of varus in tibial component coronal alignment are advised., 4,6,10

The primary aim of the present multicenter retrospective study was to determine the survivorship of a large cohort of patients implanted with a single design all-polyethylene tibial component medial UKA at a mid-term follow-up. Secondary purposes were to evaluate clinical outcomes and to investigate the reasons underlying implant failures, with specific attention to component placement and limb alignment. It was hypothesized that an all-polyethylene UKA would be a reliable prosthetic implant with superior or comparable overall survivorship to literature reports of other medial all-polyethylene implants and for metal-backed UKAs at mid-term follow-up.

\section{Materials and Methods}

This multicenter retrospective study included all patients undergoing medial all-polyethylene UKA from January 2007 to January 2013 at two different orthopaedic centers. All the operations were performed by two high-UKA volume surgeons, both of whom had at least 5 years' experience in this surgical procedure prior to the start of the case series. The present study was performed in accordance with the ethical standards of the 1964 Declaration of Helsinki, as revised in
2000. Patients were eligible for study inclusion if they had a diagnosis of primary unicompartmental OA, posttraumatic medial OA, or osteonecrosis with no prior history of joint replacement on the affected side and would comply with the follow-up schedule, with at least 1 year of follow-up. Surgical indications were homogeneous for both centers and were as follows: pain and tenderness localized to the medial joint line, active and passive knee flexion $>85$ degrees, extension lag $<10$ degrees, varus deformity $<15$ degrees as measured on long-leg standing radiographs, isolated medial compartment OA (Ahlback's grade 3-4), with no clinical evidence of patello-femoral and lateral compartment involvement. Anterior and posterior cruciate ligaments integrity was clinically checked. Exclusion criteria were as follows: prior history of osteotomy on the affected knee, coronal radiographic deformity $>15$ degrees, flexion contracture $>15$ degrees; functional incompetency of the anterior cruciate ligament (ACL) and peripheral ligaments, as well as the presence of inflammatory arthropathies or conditions determining inability to adhere to the study protocols. A total of 168 all-polyethylene tibial component UKAs (104 in Center A and 64 in Center B) were performed in 166 subjects. A total of 140 patients (142 knees, $84.5 \%$ ) were included for clinical outcome analysis (-Table $\mathbf{1}$ ).

All-polyethylene tibial component implants of the JOURNEY UNI Unicompartmental Knee System (Smith \& Nephew Inc., Memphis, TN) were implanted in all cases performing a minimally invasive mid-vastus approach. Surgery was performed according to the proposed surgical technique, as suggested by the manufacturer. The tibial coronal cut was performed respecting the tibial epiphyseal anatomy. The tibial cutting guide was adjusted in order to reproduce with the cut the angle subtended by the tibial joint line and the tibial mechanical axis on the coronal plane and the native tibial slope on the sagittal plane, as measured on the preoperative radiographs. After removal of tibial resection, the adequate trial polyethylene component was positioned, trying to use the trial component with minimum thickness $(7 \mathrm{~mm})$ and checking for flexion and extension gaps. In all knees, the femur cuts were performed aligning to the tibial cut surface. Postoperative care protocols were homogeneous for both centers. One day after surgery, blood drainage was removed, passive knee motion started, and partial weight bearing was allowed in all patients.

Table 1 Patients' demographics and characteristics after application of inclusion/exclusion criteria

\begin{tabular}{|l|l|l|l|}
\hline Parameter & Center A & Center B & Overall \\
\hline Number of patients & $80(82$ knees) & $60(60$ knees) & $140(142$ knees) \\
\hline Males/females & $47 / 33$ & $26 / 34$ & $67 / 73$ \\
\hline Right/left & $53 / 29$ & $31 / 29$ & $84 / 58$ \\
\hline Age (y) & $\begin{array}{l}67.0 \\
\text { (min. 46.3, max. 85.1) }\end{array}$ & $\begin{array}{l}68.1 \\
(\text { min. 49.2, max. 86.2) }\end{array}$ & $67.1(\min .46 .3$, max. 86.2) \\
\hline Follow-up (mo) & 61.8 (SD: 10.5, min. 14, max. 82) & $64.1(S D: 11.8$, min. 15, max. 86) & $\begin{array}{l}62.4 \\
\text { (SD: 11.2, min. 14., max. 86) }\end{array}$ \\
\hline
\end{tabular}

Abbreviations: max., maximum; min., minimum; SD, standard deviation.

Note: Values are reported as mean (standard deviations, min., and max.). 
Data analysis was performed by one independent observer in Center A and one in Center B. Observers were not involved in surgical activity. Clinical assessment was performed preoperatively at 3 and 6 months postoperatively and then yearly thereafter. Those patients who did not attend two or more consecutive clinical assessments were contacted via telephone call and asked if they had undergone revision or reoperation for any reason with tick-box options.

Pre- and postoperative visual analog $(0-10 \mathrm{~cm})$ scale (VAS) for pain assessment was collected from all patients' medical records. Knee range of motion was measured with a goniometer pre- and postoperatively at every follow-up visit. Patients were administered the five subscales (pain, symptoms, function in activities of daily living, function in sports, and quality of life) of the Knee Injury and Osteoarthritis Outcome Score ${ }^{14}$ and the Knee Society Score ${ }^{15}$ pre- and postoperatively. Implant revision for any reason was considered as failure.

Full leg anteroposterior (AP) and knee lateral weight-bearing radiographs were taken preoperatively in all patients. Postoperatively, AP and lateral weight-bearing knee radiographs were taken 1 month, at 3, 6, and 12 months postoperatively, and then yearly. Radiographic measurements were performed on the last follow-up radiographs. On the preoperative radiographs, the following angles were measured: (1) mechanical femoro-tibial angle (mFTA; - Figure 1). (2) Cartier's angle (CA), (3) anatomical femoro-tibial angle (aFTA), (4) tibial posterior slope (PS) (-Figure 2). On the postoperative knee radiographs, the following angles were measured: (1) varus/valgus alignment of the tibial component, (2) aFTA, and (3) tibial component PS (-Figure 3).

\section{Statistical Analysis}

Absolute frequency and percentage were calculated for categorical data, while means standard deviations and minimal and maximal values were used for continuous data. Statistical comparisons between paired pre- and postoperative parametric data and failed and revised radiographic outcome measures were performed using the Student's $t$ test. All tests were paired and two-sided and a $p$-value $<0.05$ was used to determine statistical significance. Survivorship analyses were performed using the Kaplan-Meier method, considering UKA revision surgery as endpoint for survivorship. The results were graphed and reported with 95\% confidence interval. Statistical analyses were performed using Wolfram Mathematica (Wolfram Research, Inc., 100 Trade Center Drive, Champaign, IL).

\section{Results}

A total of 140 patients (142 knees, 84.5\%) was included for clinical outcome analysis (-Table $\mathbf{1}$ ). The remaining 26 patients were excluded due to missing postoperative outcome data. All the 166 patients (168 knees) included for study assessment were taken into account into the survival and radiographic analysis. The average follow-up was 61.1 months (standard deviation: 11.2, minimum 14 and maximum 86 months).

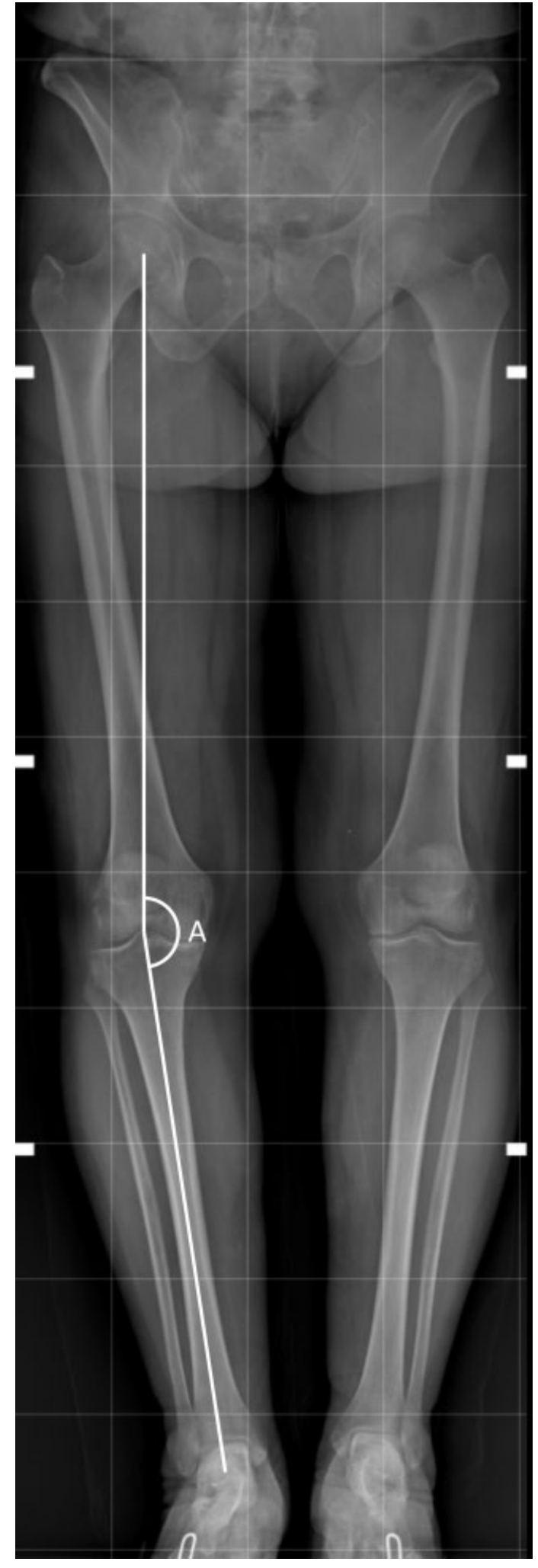

Fig. 1 Preoperative full leg standing radiograph. (A) Mechanical femorotibial angle, angle between femoral and tibial mechanical axes.

Two patients underwent UKA revision in Center $A$ and three patients in Center B, resulting in an overall revisionfree survival probability from implant of 96.5\% (CI: 91.7-98.6\%) at an average follow-up of 61.1 months (minimum 14 and maximum 86 ) of follow-up (-Figure 4). 

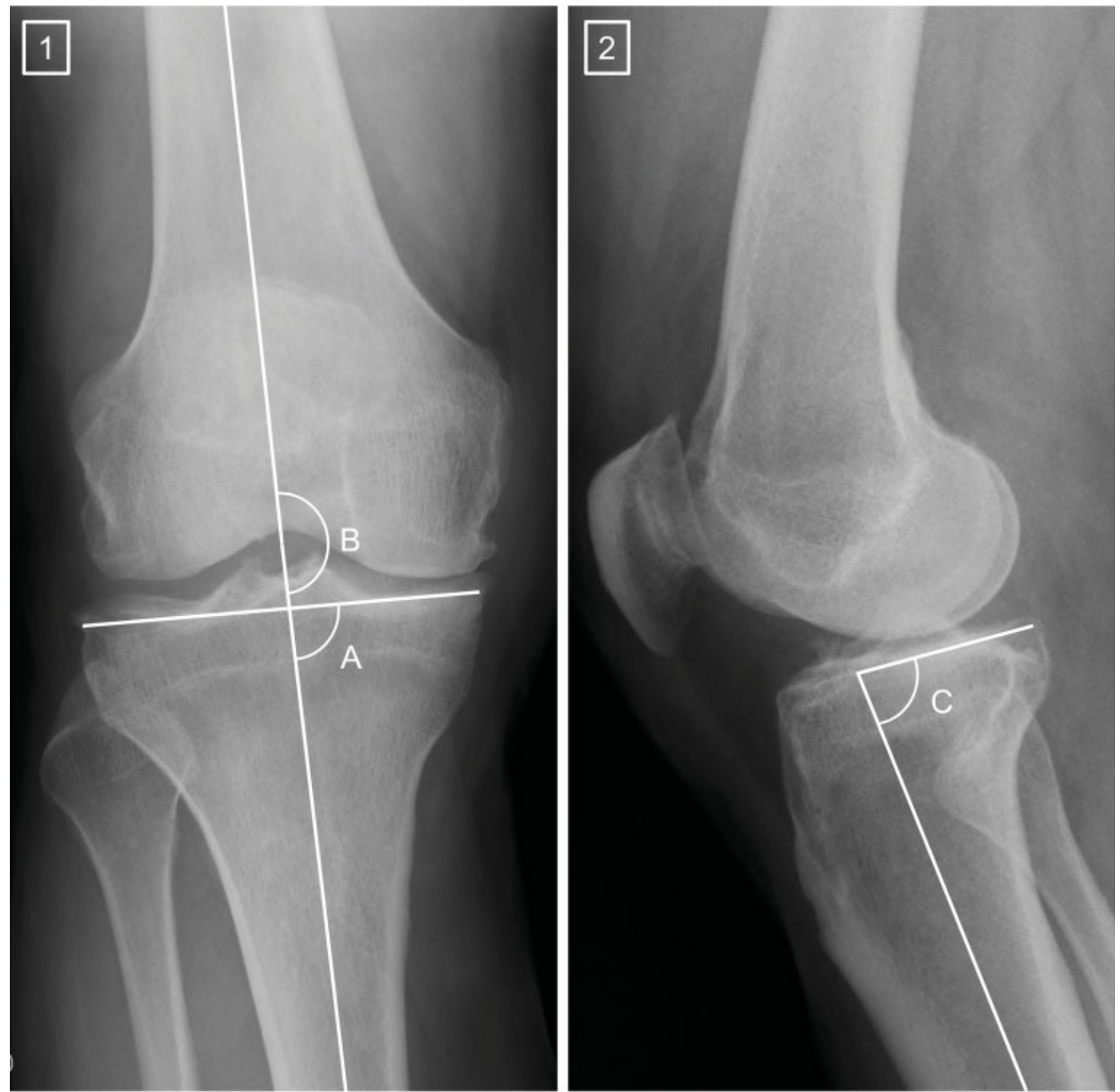

Fig. 2 Preoperative anteroposterior (1) and lateral (2) knee radiographs. (A) Cartier's angle, angle subtended between tibial joint line and tibial mechanical axis. (B) Anatomical femoro-tibial angle, angle between the femoral and tibial anatomical axes. (C) Tibial posterior slope, angle between the line perpendicular to the sagittal tibial axis and the posterior inclination of the medial tibial plateau.

Radiographic alignment assessment of failed implants was performed (-Table 2). Case 1 was revised 14 months postoperatively for persistent lateral compartment pain, with radiographic examination revealing osteoarthritis progression of the lateral compartment. Case 2 underwent revision for tibial aseptic loosening 61 months postoperatively. Case 3 underwent revision for medial compartment instability at 21 months postoperatively. In case 4 , revision was performed for incoming of medial osteonecrosis of the tibia, 30 months after surgery. Case 5 underwent revision in an external center for unexplained pain 48 months postoperatively. There were no cases of infection observed in either center. Radiographic outcome measures showed statistically significant differences in average preoperative $\mathrm{CA}(p<0.001)$ and postoperative tibial posterior slope $(p<0.001)$ between failed and not-revised UKAs ( - Table 3 ).
The clinical and functional outcomes of KOOS, KSS, ROM, and VAS all showed significant improvements over preoperative values for patients treated both at Center $A$ and Center $B$ (-Table 4).

All patients had an average radiographic varus preoperative alignment of the operated knee. No statistical significance was revealed between pre- and postoperative CA and tibial posterior slope (-Table 5 ).

\section{Discussion}

The most important finding of the present multicenter, retrospective study is the report of $96.5 \%$ Kaplan-Meier survival probability at just over 5 years of average followup of a single design all-polyethylene tibial component medial UKA at two different centers. Reports of 5-year 

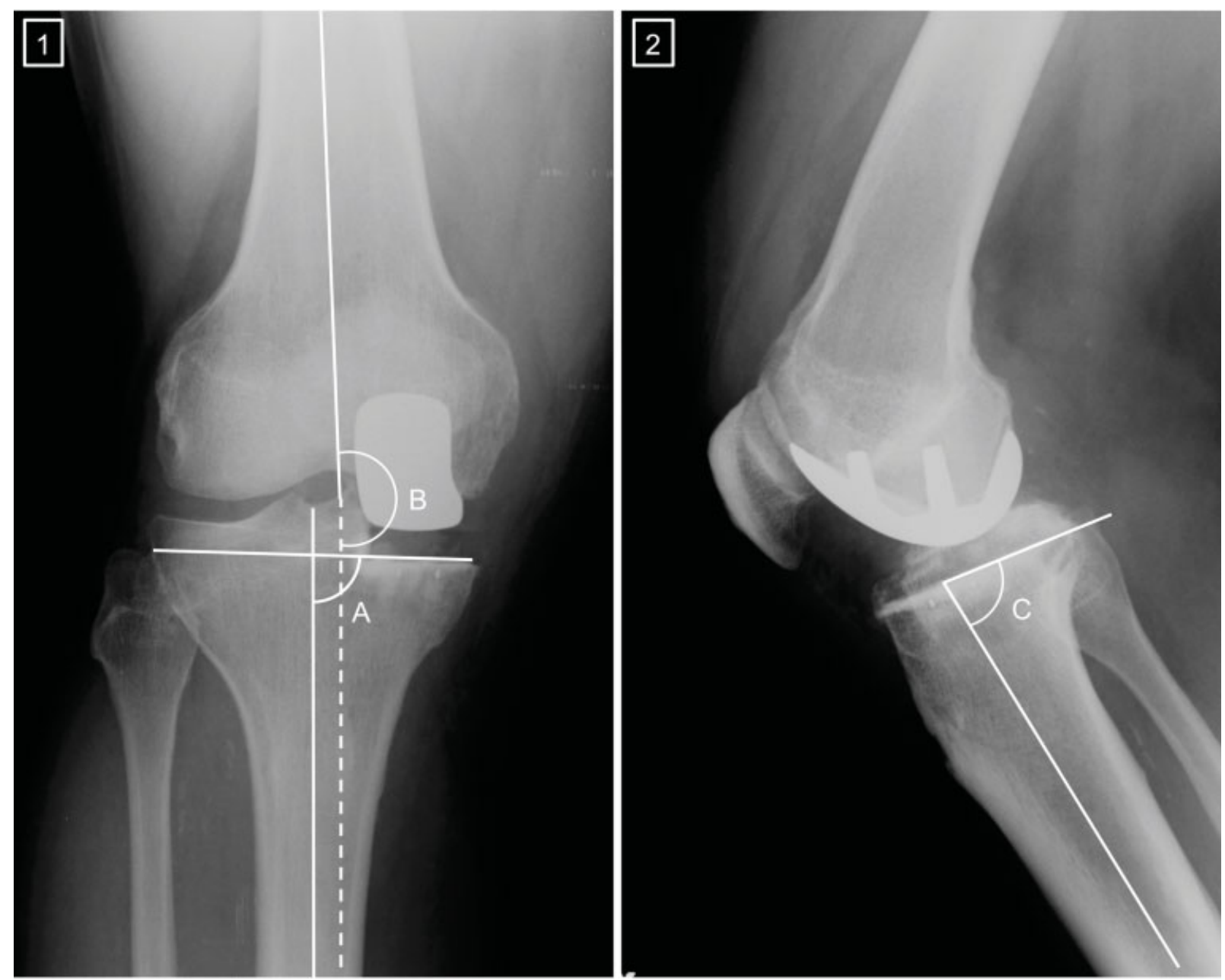

Fig. 3 Postoperative anteroposterior (1) and lateral (2) knee radiographs. (A) Varus/valgus alignment of the tibial component, angle between the tangent line to the tibial component and tibial anatomical axis. (B) Anatomical femoro-tibial angle, angle between the femoral and tibial anatomical axes. (C) Tibial posterior slope, angle between the line perpendicular to the sagittal tibial axis and the posterior inclination of the tibial component.

survival rates of medial all-polyethylene and metal-backed UKAs range from 86.0 to $96.1 \%$, and identify loosening of the tibial component, polyethylene wear, and progression of $\mathrm{OA}$ as major problems. ${ }^{9,11,13,16,17}$ Therefore, to the best of the authors' knowledge, the survivorship reported in the present study is the highest in literature for all-polyethylene tibial component UKAs so far.

A recent review analyzed causes of UKA failure, reporting that tibial component loosening (36\%) and progression of OA in the contralateral compartment (20\%) are the most common causes of UKA failure. ${ }^{11}$ Aseptic loosening has been more frequently described as an early cause of failure, whereas progression of $\mathrm{OA}$ has been more frequently reported in mid- and long-term follow-ups. Concerns have also been raised regarding the incidence of tibial loosening and subsidence in all-polyethylene tibial components, ${ }^{18}$ and the role of stiffness in unexplained pain. ${ }^{3,19}$ Different survival rates ${ }^{16,18}$ in all-polyethylene designs suggest that not all all-polyethylene designs are the same, and this may reflect component thickness. ${ }^{20}$ Components of $6 \mathrm{~mm}$ thickness have been significantly associated with increased wear and osteolysis. ${ }^{21}$ Pathological cancellous bone overload and tibial subsidence may affect thinner implants more so than thicker implants.

In the present study, five patients underwent UKA revision, two in Center A (Case 1 and Case 2) and three in Center B (Case 3, Case 4, and Case 5). One early revision (Case 1) at 14 months was performed for persistent pain on the contralateral compartment. Radiographic evaluation revealed progression of $\mathrm{OA}$ in the contralateral and patello-femoral compartment; thus, total knee arthroplasty (TKA) was performed. Another implant (Case 2) was revised at 61 months for persistent pain localized on the medial compartment. Radiographs did not demonstrate progressive radiolucency lines, but during surgical revision to TKA, tibial component aseptic loosening was noted and determined as the cause of failure. Causes of UKA failure in Center B included medial instability, which made revision necessary 21 months after surgery, and medial osteonecrosis of the tibia, which occurred 30 months after treatment. Persistent and unexplained pain was reported in one patient, making UKA revision necessary 48 months postoperatively. Only in one case (Case 2), the authors reported deviations from optimal coronal and sagittal prosthetic alignment, with a significant 


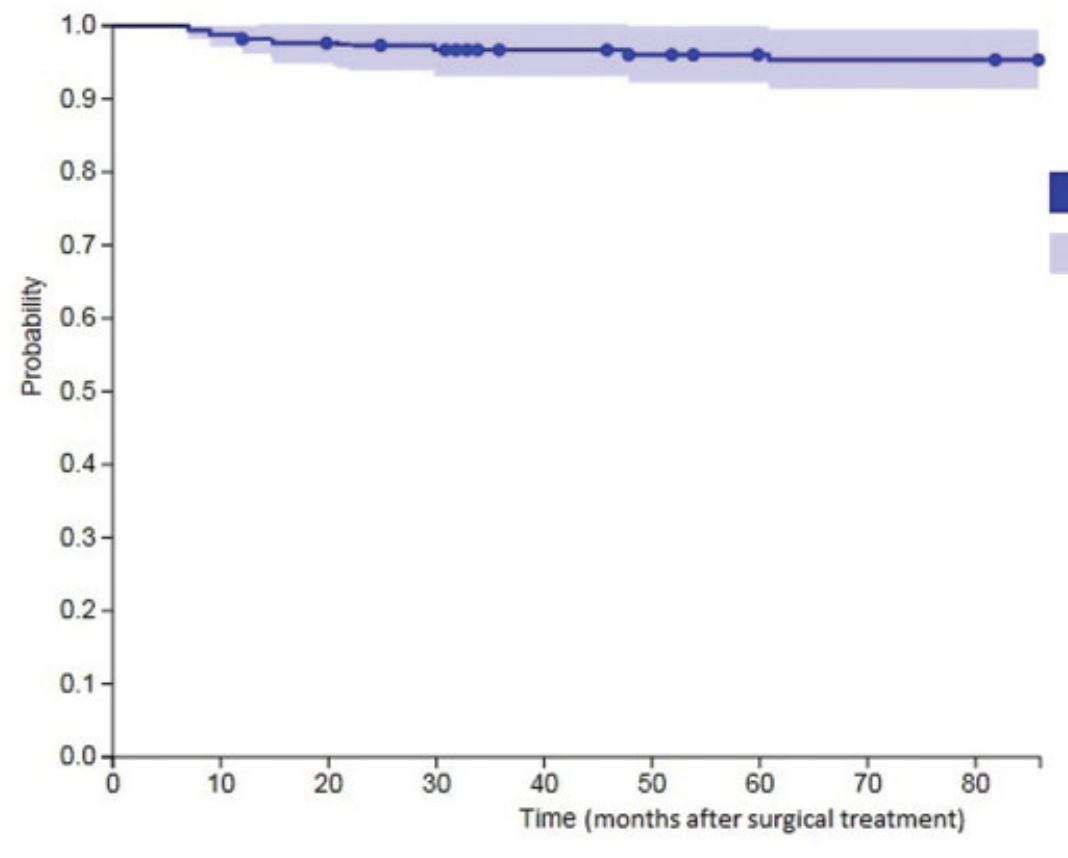

Overall survivorship: $96.5 \%$

Confidence interval: $91.7 \%-98.6 \%$

Fig. 4 Kaplan-Meier survival probability of all-polyethylene tibial component unicompartmental knee arthroplasty, considering revision as endpoint.

Table 2 Cases of unicompartmental knee arthroplasty revision in Center A and B

\begin{tabular}{|c|c|c|c|c|}
\hline Case & Radiographic parameter & Preoperatively & Postoperatively & $\begin{array}{l}\Delta \text { (preoperative - } \\
\text { postoperative aFTA) }\end{array}$ \\
\hline \multirow{4}{*}{$\begin{array}{l}\text { Case } 1 \\
\text { (revision at } \\
14 \text { months } \\
\text { postoperatively) }\end{array}$} & mFTA & -8.8 degrees & 1 & l \\
\hline & $\mathrm{aFTA}$ & -4.5 degrees & -2.0 & 2.5 \\
\hline & Posterior tibial slope & 4.4 degrees & 6.0 & 1.6 \\
\hline & Cartier angle/tibial component varus/valgus & -1.8 degrees & 1.5 & 3.3 \\
\hline \multirow{4}{*}{$\begin{array}{l}\text { Case } 2 \\
\text { (revision at } \\
61 \text { months } \\
\text { postoperatively) }\end{array}$} & $\mathrm{mFTA}$ & -8.0 & 1 & 1 \\
\hline & aFTA & -2.0 & 6.5 & 8.5 \\
\hline & Posterior tibial slope & 6.0 & 10.0 & 4.0 \\
\hline & Cartier angle/tibial component varus/valgus & -10.0 & 0.0 & 10.0 \\
\hline \multirow{4}{*}{$\begin{array}{l}\text { Case } 3 \\
\text { (revision at } \\
21 \text { months } \\
\text { postoperatively) }\end{array}$} & $\mathrm{mFTA}$ & -9.6 degrees & 1 & I \\
\hline & aFTA & -6.0 degrees & -2.5 & 3.5 \\
\hline & Posterior tibial slope & 5.0 degrees & 5.0 & 0.0 \\
\hline & Cartier angle/tibial component varus/valgus & -2.0 degrees & -1.5 degrees & 0.5 \\
\hline \multirow{4}{*}{$\begin{array}{l}\text { Case } 4 \\
\text { (revision at } \\
30 \text { months } \\
\text { postoperatively) }\end{array}$} & mFTA & -8.5 & 1 & I \\
\hline & aFTA & -4.0 & -1.0 & 3.0 \\
\hline & Posterior tibial slope & 3.0 & 3.0 & 0.0 \\
\hline & Cartier angle/tibial component varus/valgus & -1.0 & -1.0 & 0.0 \\
\hline \multirow{4}{*}{$\begin{array}{l}\text { Case } 5 \\
\text { (revision at } \\
48 \text { months } \\
\text { postoperatively }\end{array}$} & mFTA & -8.3 & 1 & 1 \\
\hline & aFTA & -4.0 & -2.5 & 1.5 \\
\hline & Posterior tibial slope & 5.0 & 4.5 & 0.5 \\
\hline & Cartier angle/tibial component varus/valgus & 0.0 & -2.0 & 2.0 \\
\hline
\end{tabular}

Abbreviations: aFTA, anatomical femoro-tibial angle; mFTA, mechanical femoro-tibial angle.

Note: Positive values are suggestive of valgus alignment, negative values of varus alignment. 
Table 3 Preoperative and postoperative radiographic alignment of failed and surviving implants

\begin{tabular}{|l|l|l|l|}
\hline & $\begin{array}{l}\text { Failed implants } \\
(\boldsymbol{n}=\mathbf{5})\end{array}$ & $\begin{array}{l}\text { Surviving implants } \\
(\boldsymbol{n}=\mathbf{1 3 5})\end{array}$ & $\boldsymbol{p}$-Value \\
\hline Preoperative aFTA & -4.1 degrees (SD: 1.4) & 0.7 degrees (SD: 2.8) & n.s. \\
\hline Postoperative aFTA & -1.6 degrees (SD: 1.1$)$ & 2.8 degrees (SD: 2.9) & n.s. \\
\hline Cartier's angle & -3.0 degrees (SD: 4.0) & -2.1 degrees (SD: 2.2) & $<0.001$ \\
\hline Tibial component varus/valgus & 0.0 degrees (SD: 1.5) & -1.7 degrees (SD: 1.3) & n.s. \\
\hline Preoperative tibial posterior slope & 4.7 degrees (SD: 1.1) & 7.6 degrees (SD: 1.4$)$ & n.s. \\
\hline Postoperative tibial posterior slope & 5.7 degrees (SD: 2.6) & 7.2 degrees (SD: 1.9$)$ & $<0.001$ \\
\hline
\end{tabular}

Abbreviations: aFTA, anatomical femoro-tibial angle; n.s., not statistically significant; SD, standard deviation.

Note: Values are reported as mean and standard deviations. Positive values are suggestive of valgus alignment, negative values of varus alignment.

Table 4 Patients' reported outcome measures in Center A and B

\begin{tabular}{|l|l|l|l|l|}
\hline Parameter & Preoperative & Postoperative & $\Delta$ & $p$-Value \\
\hline Range of motion & $\begin{array}{l}\text { 99.3 degrees } \\
\text { (SD: 12.2, min. 90, max. 125) }\end{array}$ & $\begin{array}{l}128.2 \text { degrees } \\
\text { (SD: 10.3, min. 90, max. 135) }\end{array}$ & $\begin{array}{l}28.9 \\
\text { degrees }\end{array}$ & $<0.0001$ \\
\hline VAS (0-10) & 5.3 (SD: 1.2, min. 3, max. 8) & 1.3 (SD: 1.6, min. 0, max. 6) & 4.0 & $<0.0001$ \\
\hline KOOS & & & & \\
\hline Pain & 39.0 (SD: 17.0) & 85.7 (SD: 17.3) & 46.7 & $<0.001$ \\
\hline Symptoms & 47.3 (SD: 20.9) & 89.5 (SD: 14.3$)$ & 42.2 & $<0.001$ \\
\hline $\begin{array}{l}\text { Function in activities } \\
\text { of daily living }\end{array}$ & 40.8 (SD: 19.3) & 87.2 (SD: 7.4$)$ & 46.4 & $<0.001$ \\
\hline $\begin{array}{l}\text { Function in sports } \\
\text { and recreation }\end{array}$ & 26.0 (SD: 17.0$)$ & 67.0 (SD: 27.0$)$ & 41.0 & $<0.001$ \\
\hline Quality of Life & 24.0 (SD: 10.0$)$ & 77.6 (SD: 26.0) & 53.6 & $<0.001$ \\
\hline Total & 35.4 (SD: 17.3$)$ & 81.4 (SD: 19.4$)$ & 46.0 & $<0.001$ \\
\hline KSS & & & & \\
\hline Knee & 51.0 (SD: 8.5$)$ & 90.3 (SD: 12.3$)$ & 39.3 & $<0.00001$ \\
\hline Function & 41.7 (SD: 12.2$)$ & 89.2 (SD: 15.2$)$ & 47.5 & $<0.00001$ \\
\hline
\end{tabular}

Abbreviations: KOOS, Knee Injury and Osteoarthritis Outcome Score; KSS, Knee Society Score; max., maximum; min., minimum; SD, standard deviation; VAS, Visual Analogue Scale.

Note: Values are reported as mean (standard deviations, min., and max.).

correction of the preoperative CA ( -10 degrees) to a neutral alignment of the tibial component. No other alignment defects were noted in revised patients in any center.

Although there are no direct correlations between alignment deviations and clinical results, current literature underlines that deviations in coronal tibial component alignment can lead to polyethylene wear and early loosening. ${ }^{10,22-24}$ As noted by Hernigou et $\mathrm{al}^{21}$ a slight undercorrection of the preoperative tibial varus deformity is likely to be performed. Others recommend avoiding tibial coronal alignment higher than 5 to 6 degrees of varus and sagittal slope of more than 7 degrees, ${ }^{4,6,13,25}$ in particular when performing all-polyethylene tibial component UKAs. ${ }^{5}$ In the present study, medial tibial plateau alignment on both coronal and sagittal plane was preserved, with no statistically significant differences reported between radiographic preoperative $\mathrm{CA}$ and postoperative tibial component angle, nor for preoperative and postoperative tibial posterior slope.
Postoperative aFTA was less than 6 degrees of valgus in all patients, with the exception of a single case that underwent revision to TKA after 61 months (case 2, Center A).

The excellent mean postoperative KOOS scores and the statistically significant difference between pre- and postoperative mean clinical subscales, highlight the efficacy of the examined all-polyethylene tibial component UKA in restoring knee function. The mean "Function in Sports and Recreation" KOOS subscale did not reach excellent results. This specific result is explained by patient comorbidities, including $\mathrm{OA}$ on the contralateral knee and other age-related physical impairment.

The present study has several limitations. First, postoperative knee mechanical alignment was not available for most of the patients, as weight-bearing radiographs of the lower limbs are frequently not requested for postoperative implant evaluation. However, studies have demonstrated that standard-view knee radiographs are reliable 
Table 5 Mean pre- and postoperative radiographic alignment of patients in Center A, Center B, and cumulative data

\begin{tabular}{|c|c|c|c|c|}
\hline & $\begin{array}{l}\text { Center A } \\
(n=82)\end{array}$ & $\begin{array}{l}\text { Center B } \\
(n=60)\end{array}$ & $\begin{array}{l}\text { Overall } \\
(n=142)\end{array}$ & $p$-Value \\
\hline Preoperative mFTA & -6.3 degrees (SD: 3.2) & -6.9 degrees (SD: 2.6) & -6.6 degrees (SD: 2.9) & \\
\hline Preoperative aFTA & 0.4 degrees (SD: 2.8 ) & 0.9 degrees (SD: 3.0 ) & 0.6 degrees (SD: 2.9) & \\
\hline Postoperative aFTA & 3.1 degrees (SD: 3.3) & 2.5 degrees (SD: 2.8 ) & 2.8 degrees (SD: 3.0 ) & \\
\hline$\Delta$ (preoperative-postoperative aFTA) & & & 2.2 degrees (SD: 5.9 ) & $<0.001$ \\
\hline Cartier's angle & -2.4 degrees (SD: 3.0 ) & -1.4 degrees (SD: 1.1 ) & -2.1 degrees (SD: 2.1 ) & \\
\hline Tibial component varus/valgus & -2.1 degrees (SD: 1.8$)$ & -1.1 degrees (SD: 1.0$)$ & -1.7 degrees (SD: 1.4$)$ & \\
\hline $\begin{array}{l}\Delta \text { (Cartier's angle-tibial } \\
\text { component varus/valgus) }\end{array}$ & & & 0.4 (SD: 3.5$)$ & n.s. \\
\hline Preoperative posterior tibial slope & 7.0 degrees (SD: 3.0 ) & 8.2 degrees (SD: 1.9 ) & 7.5 degrees (SD: 1.6$)$ & \\
\hline Postoperative posterior tibial slope & 6.4 degrees (SD: 2.0) & 8.3 degrees (SD: 1.9 ) & 7.2 degrees (SD: 2.0 ) & \\
\hline $\begin{array}{l}\Delta \text { (preoperative-postoperative } \\
\text { posterior tibial slope) }\end{array}$ & & & 0.3 degrees (SD: 3.6$)$ & n.s. \\
\hline
\end{tabular}

Abbreviations: aFTA, anatomical femoro-tibial angle; mFTA, mechanical femoro-tibial angle, n.s., not statistically significant; SD, standard deviation. Note: Values are reported as mean and standard deviations. Positive values are suggestive of valgus alignment, negative values of varus alignment.

instruments to evaluate postoperative knee alignment. ${ }^{26}$ Second, the sample of patients is rather small, and only five cases of failure were available for assessment. Third, body mass index (BMI) and its effect on UKA outcome were not considered in the present investigation. Increased BMI has recently been correlated with increased risks of failure in all-polyethylene tibial components UKA; however, none of the revised patients had BMI higher than 30. Follow-up length is heterogeneous, but it is the authors' hope that further studies with longer follow-ups would better evaluate effects of all-polyethylene tibial component positioning on UKA failure.

\section{Conclusion}

All-polyethylene tibial component UKAs provided satisfactory clinical and functional outcome, with a low number of UKA revision in the early- and mid-term follow-up. Aseptic tibial loosening, which UKA failure has often been accounted for in all-polyethylene tibial components UKA, was reported only in one case. This demonstrates that appropriate positioning and polyethylene insert thickness are factors to be taken into account when choosing an all-polyethylene tibial component UKA.

\section{Conflict of Interest}

None declared.

\section{References}

1 Furnes O, Espehaug B, Lie SA, Vollset SE, Engesaeter LB, Havelin LI. Failure mechanisms after unicompartmental and tricompartmental primary knee replacement with cement. J Bone Joint Surg Am 2007;89(03):519-525

2 Gladnick BP, Nam D, Khamaisy S, Paul S, Pearle AD. Onlay tibial implants appear to provide superior clinical results in robotic unicompartmental knee arthroplasty. HSS J 2015;11(01):43-49
3 Small SR, Berend ME, Ritter MA, Buckley CA, Rogge RD. Metal backing significantly decreases tibial strains in a medial unicompartmental knee arthroplasty model. J Arthroplasty 2011;26(05): 777-782

4 Bruni D, Akkawi I, Iacono F, et al. Minimum thickness of all-poly tibial component unicompartmental knee arthroplasty in patients younger than 60 years does not increase revision rate for aseptic loosening. Knee Surg Sports Traumatol Arthrosc 2013; 21(11):2462-2467

5 Chatellard R, Sauleau V, Colmar M, Robert H, Raynaud G, Brilhault J; Société d'Orthopédie et de Traumatologie de l'Ouest (SOO). Medial unicompartmental knee arthroplasty: does tibial component position influence clinical outcomes and arthroplasty survival? Orthop Traumatol Surg Res 2013;99(04):S219-S225

6 Lustig S, Paillot JL, Servien E, Henry J, Ait Si Selmi T, Neyret P. Cemented all polyethylene tibial insert unicompartimental knee arthroplasty: a long term follow-up study. Orthop Traumatol Surg Res 2009;95(01):12-21

7 Koh IJ, Suhl KH, Kim MW, Kim MS, Choi KY, In Y. Use of Allpolyethylene Tibial Components in unicompartmental knee arthroplasty increases the risk of early failure. J Knee Surg 2017;30(08):807-815

8 Heyse TJ, Khefacha A, Peersman G, Cartier P. Survivorship of UKA in the middle-aged. Knee 2012;19(05):585-591

9 Hawi N, Plutat J, Kendoff D, et al. Midterm results after unicompartmental knee replacement with all-polyethylene tibial component: a single surgeon experience. Arch Orthop Trauma Surg 2016;136(09):1303-1307

10 Pandit H, Jenkins C, Gill HS, Barker K, Dodd CA, Murray DW. Minimally invasive Oxford phase 3 unicompartmental knee replacement: results of 1000 cases. J Bone Joint Surg Br 2011;93 (02):198-204

11 van der List JP, Zuiderbaan HA, Pearle AD. Why do medial unicompartmental knee arthroplasties fail today? J Arthroplasty 2016;31(05):1016-1021

12 Kim KT, Lee S, Lee JI, Kim JW. Analysis and treatment of complications after unicompartmental knee arthroplasty. Knee Surg Relat Res 2016;28(01):46-54

13 Zambianchi F, Digennaro V, Giorgini A, et al. Surgeon's experience influences UKA survivorship: a comparative study between allpoly and metal back designs. Knee Surg Sports Traumatol Arthrosc 2015;23(07):2074-2080 
14 Roos EM, Roos HP, Lohmander LS, Ekdahl C, Beynnon BD. Knee Injury and Osteoarthritis Outcome Score (KOOS)-development of a self-administered outcome measure. J Orthop Sports Phys Ther 1998;28(02):88-96

15 Insall JN, Dorr LD, Scott RD, Scott WN. Rationale of the Knee Society clinical rating system. Clin Orthop Relat Res 1989;(248):13-14

16 Bruni D, Gagliardi M, Akkawi I, et al. Good survivorship of allpolyethylene tibial component UKA at long-term follow-up. Knee Surg Sports Traumatol Arthrosc 2016;24(01):182-187

17 Manzotti A, Cerveri P, Pullen C, Confalonieri N. A flat all-polyethylene tibial component in medial unicompartmental knee arthroplasty: a long-term study. Knee 2014;21(Suppl 1):S20-S25

18 Hutt JR, Farhadnia P, Massé V, LaVigne M, Vendittoli PA. A randomised trial of all-polyethylene and metal-backed tibial components in unicompartmental arthroplasty of the knee. Bone Joint J 2015;97-B(06):786-792

19 Scott CE, Eaton MJ, Nutton RW, Wade FA, Pankaj P, Evans SL. Proximal tibial strain in medial unicompartmental knee replacements: a biomechanical study of implant design. Bone Joint J 2013;95-B(10): 1339-1347

20 Scott CE, Eaton MJ, Nutton RW, Wade FA, Evans SL, Pankaj P. Metalbacked versus all-polyethylene unicompartmental knee arthro- plasty: Proximal tibial strain in an experimentally validated finite element model. Bone Joint Res 2017;6(01):22-30

21 Hernigou P, Poignard A, Filippini P, Zilber S. Retrieved unicompartmental implants with full PE tibial components: the effects of knee alignment and polyethylene thickness on creep and wear. Open Orthop J 2008;2:51-56

22 Clarius M, Hauck C, Seeger JB, Pritsch M, Merle C, Aldinger PR. Correlation of positioning and clinical results in Oxford UKA. Int Orthop 2010;34(08):1145-1151

23 Collier MB, Eickmann TH, Sukezaki F, McAuley JP, Engh GA. Patient, implant, and alignment factors associated with revision of medial compartment unicondylar arthroplasty. J Arthroplasty 2006;21(06, Suppl 2):108-115

24 Price AJ, Svard U. A second decade lifetable survival analysis of the Oxford unicompartmental knee arthroplasty. Clin Orthop Relat Res 2011;469(01):174-179

25 Sawatari T, Tsumura H, Iesaka K, Furushiro Y, Torisu T. Threedimensional finite element analysis of unicompartmental knee arthroplasty-the influence of tibial component inclination. J Orthop Res 2005;23(03):549-554

26 Fang D, Ritter MA. Malalignment: forewarned is forearmed. Orthopedics 2009;32(09):orthosupersite.com/view.asp?rID=42850 